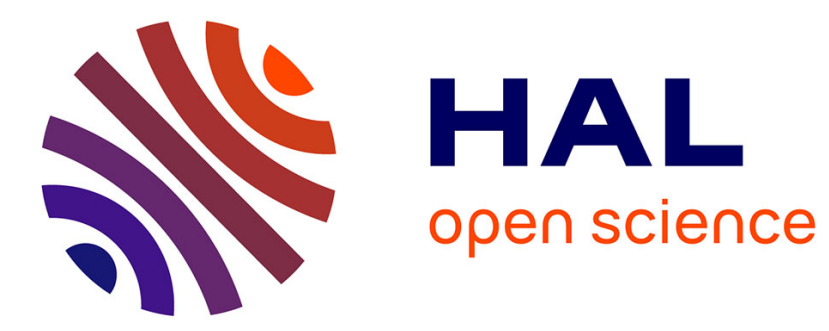

\title{
Akhmediev breathers as ultra-wideband pulses
}

\author{
Bastien Varlot, Yanne Kouomou Chembo, Christophe Finot
}

\section{To cite this version:}

Bastien Varlot, Yanne Kouomou Chembo, Christophe Finot. Akhmediev breathers as ultra-wideband pulses. Microwave and Optical Technology Letters, 2014, 56 (3), pp.664-667. 10.1002/mop.28174. hal-00878042

\section{HAL Id: hal-00878042 \\ https://hal.science/hal-00878042}

Submitted on 29 Oct 2013

HAL is a multi-disciplinary open access archive for the deposit and dissemination of scientific research documents, whether they are published or not. The documents may come from teaching and research institutions in France or abroad, or from public or private research centers.
L'archive ouverte pluridisciplinaire HAL, est destinée au dépôt et à la diffusion de documents scientifiques de niveau recherche, publiés ou non, émanant des établissements d'enseignement et de recherche français ou étrangers, des laboratoires publics ou privés. 


\title{
Akhmediev breathers as ultra-wideband pulses
}

\author{
B. Varlot $^{1}$, Y. Chembo ${ }^{2}$ and C. Finot ${ }^{1, *}$ \\ 1. Laboratoire Interdisciplinaire CARNOT de Bourgogne, \\ UMR 6303 CNRS-Université de Bourgogne, \\ 9 Av. A. Savary, BP 47870,21078 DIJON Cedex, FRANCE \\ 2. FEMTO-ST Institute \\ UMR 6174 CNRS-Université de Franche-Comté, \\ 16 Route de Gray, 25030 BESANCON Cedex, FRANCE. \\ *christophe.finot@u-bourgogne.fr
}

\begin{abstract}
We analytically calculate and discuss the radio-frequency spectrum of the socalled Akhmediev breathers, a class of nonlinear solutions of the nonlinear Schrodinger equation that governs the propagation in a single mode optical fiber. We propose a practical application of $A B s$ to the field of ultra-wideband pulse generation.
\end{abstract}

Key words : nonlinear optics, ultra-wideband, fiber optics 


\section{Introduction}

Solitons on finite background (SFB) are solutions of the nonlinear Schrödinger equation (NLSE) that are presently stimulating a renewed and widespread attention in nonlinear science. Even if their analytical expressions have been proposed for more than 25 years [1, 2], these nonlinear structures have been only recently identified as promising prototypes of the infamous oceanic rogue waves $[3,4]$. Indeed, due to the periodic exchange of energy between a continuous background and a localized structure, an initially small perturbation can exponentially grow into a brief and intense spike before disappearing. With the emergence of the field of optical rogue waves, theoretical and experimental works have confirmed that optical fibers represent an ideal testbed to easily generate and study SFB : Peregrine soliton [5], Akhmediev breathers (ABs) [6], or Kuznetsov-Ma (KM) solitons [7] have indeed been demonstrated taking advantage of widely available components for ultrafast optics. Connections with modulation instability [8], supercontinuum generation [9] and generation of ultrashort structures [10,11] have recently been suggested but at this stage, no solution has been designed to take advantage of the specific features of the SFB.

In this context, it sounds appealing to investigate the potential of Akhmediev breathers in the field of optical ultra-wideband pulse generation. Indeed, whereas the temporal intensity or phase profiles $[5,12]$, the associated optical spectra [6] or the longitudinal recurrence [12, 13], have already been the subject of cautious theoretical and experimental works, the radio-frequency $(\mathrm{RF})$ spectrum has not been exploited yet. 
We analytically calculate in this contribution the RF spectrum of $A B s$ and demonstrate that they exhibit an ultra-wideband spectrum whose central frequency depends on the system parameters. This letter will be organized as follows. After having recalled the specific features of $A B s$, we provide the analytical expression of their RF spectrum. The evolution of the central frequency of this spectrum according to the parameters of the $A B$ is then discussed. Finally, we compare the RF spectrum of ABs with spectra of usual Gaussian singlets and doublets for practical application to ultra-wideband pulse generation.

\section{Akhmediev breathers}

The propagation of light in optical fibers is described by the NLSE :

$$
i \frac{\partial \psi}{\partial z}=i \frac{\beta_{2}}{2} \frac{\partial^{2} \psi}{\partial t^{2}}+i \gamma|\psi|^{2} \psi-\frac{\alpha}{2} \psi
$$

where $z$ and $t$ denote the distance and retarded time (in the frame travelling at the group-velocity) coordinates; $\beta_{2}$ and $\gamma$ are the group velocity dispersion and the nonlinear Kerr coefficient, and $\psi(t, z)$ is the field envelope. In order to simplify the discussion, it is interesting to introduce the normalized distance $\xi$ related to the dimensional distance via $\xi=z / L_{N L}$ with the nonlinear length $L_{N L}=1 /\left(\gamma P_{0}\right)$ for an initial plane wave of power $P_{0}$.

$A B$ is a class of solutions of the NLSE whose general expression (neglecting a term of propagating phase) reads as : 


$$
\psi(t, \xi)=\sqrt{P_{0}} \frac{(1-4 a) \cosh (b \xi)+i b \sinh (b \xi)+\sqrt{2 a} \cos \left(\omega_{\text {mod }} t\right)}{\sqrt{2 a} \cos \left(\omega_{\text {mod }} t\right)-\cosh (b \xi)}
$$

The parameters $a$ and $b$ are the normalized perturbation frequency and instability growth rate, and in dimensional units are given by $2 a=1-\left(\omega_{\text {mod }} / \omega_{c}\right)^{2}$ and $b=\sqrt{8 a(1-2 a)}$. Here $\omega_{\text {mod }}$ denotes the dimensional modulation frequency in the range $0<\omega_{\text {mod }}<\omega_{c}$, where $\omega_{c}^{2}=4 \gamma P_{0} /\left|\beta_{2}\right| . a$ is comprised between 0 and 0.5 , the value $a=0.5$ leading to the solution known as the Peregrine soliton.

At the point of maximum compression (i.e. at $\xi=0$ ), the compressed field is given by :

$$
\psi(t, \xi=0)=\sqrt{P_{0}} \frac{(1-4 a)+\sqrt{2 a} \cos \left(\omega_{\bmod } t\right)}{\sqrt{2 a} \cos \left(\omega_{\bmod } t\right)-1}
$$

We compare the temporal intensity profiles obtained for a selection of three values of $a(0.25,0.375$ and 0.47$)$. The value $a=0.25$ leads to the maximum of gain of the modulation instability process whereas 0.47 represents the highest value of $a$ that has been to date experimentally reported [5]. The various examples are plotted on panels (1) of Fig. $1(a=0.25,0.375$ and 0.47 correspond to subplots $\mathrm{a}, \mathrm{b}$ and $\mathrm{c}$ respectively). One can then clearly notice ultrashort and intense spikes of light lying over a continuous background as well as the increasing compression experienced for higher values of $a$.

The spectrum $s(\omega)$ of the AB (i.e. the Fourier transform of Eq. (2), $\omega$ being the angular frequency) is well documented [6] and exhibits a typical 
triangular shape when plotted on a logarithmic scale, highlighting the decrease of the frequency components. Due to the intrinsic temporal periodicity of $\mathrm{ABs}$, the resulting spectrum is a comb with the amplitude $s_{n}$ of the different frequency components given by [6] (here, factors of constant amplitude and phase are ignored):

$$
\left\{\begin{array}{l}
s_{0}(\xi)=\frac{i b \sinh (b \xi)+p^{2} \cosh (b \xi)}{\sqrt{\cosh ^{2}(b \xi)-2 a}}-1 \\
s_{n}(\xi)=\frac{i b \sinh (b \xi)+p^{2} \cosh (b \xi)}{\sqrt{\cosh ^{2}(b \xi)-2 a}}\left[\frac{\cosh (b \xi)-\sqrt{\cosh ^{2}(b \xi)-2 a}}{\sqrt{2 a}}\right]^{n}
\end{array}\right.
$$

with $n \geq 1$ and the parameter $p$ being $p=\sqrt{2} \omega_{\bmod } / \omega_{c}$.

By stating

$$
\left\{\begin{array}{l}
r=\frac{i b \sinh (b \xi)+p^{2} \cosh (b \xi)}{\sqrt{\cosh ^{2}(b \xi)-2 a}} \\
q=\frac{\cosh (b \xi)-\sqrt{\cosh ^{2}(b \xi)-2 a}}{\sqrt{2 a}},
\end{array}\right.
$$

one can rewrite Eq. (4) into the following form :

$$
\left\{\begin{array}{l}
s_{0}=r-1 \\
s_{n}=r q^{|n|}
\end{array}\right.
$$

Results are illustrated on panels (2) of Fig. 1 with the optical spectra (i.e. the square of the various coefficients (6)) obtained for different values of $a$. The continuously decreasing amplitude of the $s_{n}$ coefficients is confirmed, with a spectral extent increasing with higher values of $a$. We can note that the specific value of $a=0.375$ leads to a cancelling of the central spectral component of the field. 


\section{Radio frequency spectrum of $A B s$}

The RF spectrum $S(\omega)$ of ABs (i.e. the Fourier transform of the square of Eq.

(2)) has been to date fully unexplored and can be derived from Eqs (6) taking into account that $S(\omega)=s(\omega) * \bar{s}(\omega)$ with $*$ being the convolution product and $\bar{s}$ being the complex conjugate of $s$. It leads to the general amplitude of the sideband $n \geq 1 \quad(\operatorname{Re}(r)$ being the real part of $r)$ :

$$
S_{n}=q^{|n|}\left\{|r|^{2}\left(|n|+\frac{1+q^{2}}{1-q^{2}}\right)-2 \operatorname{Re}(r)\right\}
$$

so that the overall envelope of the RF spectrum is given by :

$$
S(\omega)=q^{\mid \frac{\omega}{\omega_{\text {mod }}}}\left\{|r|^{2}\left(\left|\frac{\omega}{\omega_{\text {mod }}}\right|+\frac{1+q^{2}}{1-q^{2}}\right)-2 \operatorname{Re}(r)\right\}
$$

The RF spectrums and the associated envelope corresponding to the fields investigated in the previous section are plotted on Fig. 2. Compared to the simple algebraic decay of the optical spectrum tails, the RF spectrum undergoes a more complex evolution with frequency. The most important feature that can be made out is that the RF spectrum does not exhibit a monotonic decrease with increasing frequency: the RF envelope of the ABs has a local maximum which frequency depends on the value of $a$. For $a=$ 0.25 , the maximum of the RF spectrum is located at $\omega / \omega_{\bmod }=1$, whereas for $a$ $=0.375$ and 0.47 , the maximum is shifted to the second and fourth spectral components respectively.

One question that may arise is the influence of the propagation distance $\xi$. Figue $3(\mathrm{a})$ where the longitudinal evolution of the RF spectrum envelope for $a=0.47$ is plotted outlines that the most significant shift is obtained at the point of maximum compression. From Eq. (8), one can 
evaluate more quantitatively the frequency $\omega_{\max }$ at which the envelope is maximum :

$$
\frac{\omega_{\max }}{\omega_{\mathrm{mod}}}=-\frac{1}{\ln q}-\frac{1+q^{2}}{1-q^{2}}+\frac{2}{r}
$$

which can be simplified at the point of maximum of compression (where

$$
\begin{aligned}
r=2 \sqrt{1-2 a} \text { and } q & \left.=\frac{1-\sqrt{1-2 a}}{\sqrt{2 a}}\right) \text { into : } \\
\frac{\omega_{\max }}{\omega_{\mathrm{mod}}}(\xi=0) & =\frac{1}{\ln (2 a) / 2-\ln (1-\sqrt{1-2 a})}-\frac{1-\sqrt{1-2 a}}{2 a-1+\sqrt{1-2 a}}+\frac{1}{\sqrt{1-2 a}}
\end{aligned}
$$

Results are plotted in Fig. 3(b) and clearly points out that the central frequency continuously increases for increasing values of $a$.

\section{Comparison with simple shapes of ultra-wideband pulses}

The generation of an ultrashort pulse train with a maximum of the RF spectrum shifted towards high frequency is a feature of interest in the context of ultra-wideband pulse generation [14]. Indeed, as the experimental setup involved in the generation of ABs is remarkably simple and can be easily scaled to repetitions rates of several tens of $\mathrm{GHz}$ [5], it is worthy to compare the performance of the $A B$ with the spectra of widely used pulse trains such as Gaussian singlet or doublets.

We have therefore considered here an $A B$ at the point of maximum compression with a value of $a$ of 0.47 . The resulting RF spectrum is compared on Fig. 4(a) with Gaussian singlets and doublets having the same central frequency. The RF spectrum of an $A B$ is slightly broader compared with the 
singlet, but significantly larger than the spectrum of a doublet. Corresponding intensity profiles are compared on Fig. 4(b) and show that obtaining similar maximum frequencies of the RF spectrum requires a shorter structure in the case of an ABs. For the same average power, the ABs exhibits a higher peak level, so that the pulsed part better distinguishes from the continuous background.

\section{Conclusions}

We have analytically calculated the RF spectrum of $A B s$ and highlighted a significant feature of the soliton over finite background: contrary to their optical spectrum, their RF spectrum does not exhibit a monotonic evolving shape and the location of the maximum of this spectrum can be directly related to the $a$ parameter that takes both the fiber and light properties into account. To exploit this intrinsic property of the ABs can be of interest for ultra-wideband pulse generation. Even if the performance of this pulse shape does not seem to compare favorably with usual Gaussian singlets and doublets, the ease of implementation of the experimental generation may constitute an attractive architecture if very high repetition rates are targeted. The present discussion has been carried out in the context of passive fiber generation but breathers can also be observed in microresonators [15], paving the way to the all-optical and cost-effective generation on a compact optical chip. The proposed approach further stress all the benefits that can be obtained from the 
controlled shaping occurring in a medium presenting dispersion and Kerr nonlinearity [16].

\section{Acknowledgements:}

We thank Kamal Hammani, Bertrand Kibler, Julien Fatome and G. Millot for stimulating discussions. We acknowledge the financial support of the Conseil Regional de Bourgogne (Pari Photcom) and the Agence Nationale de la Recherche (project SO FAST, ANR-11-EMMA-0005 and OPTIROC, ANR-12BS04-0011). We also thank the funding of the Labex ACTION program (ANR11-LABX-01-01).I 


\section{Figure captions:}

Fig. 1 Train of optical breathers for different values of $a: a=0.25,0.375$ and 0.47 are plotted in panels $a, b$ and $c$ respectively. The temporal intensity profiles at the point of maximum compression (Eq. (3)) are plotted in panels 1 whereas the optical spectra (square of Eq. (4)) are displayed in panels 2. Time is normalized with respect to the period of the pulse train whereas frequency is normalized with respect to the repetition rate of the periodic signal.

Fig. 2 RF spectra obtained for different values of $a=0.25,0.375$ and 0.47 (subplots a, b, c respectively). The numerical Fourier transform of Eq. (3) (black line) is compared with the analytical predictions of Eq. (7) (solid black circles) and with the envelope of the RF spectrum (grey solid line, Eq. (8)).

Fig. 3 (a) Longitudinal evolution of the envelope of the RF spectrum for $a=$ 0.47. (b) Evolution of the frequency of the maximum of the RF spectrum with respect to the parameter $a$ at the point of maximum compression according to Eq. (10).

Fig. 4 (a) Comparison of the envelopes of the RF spectrum of a train of ABs (solid black line, $a=0.47$ ) with the RF spectrum of a Gaussian singlet (dashdotted line) and Gaussian doublet (dashed grey line) having similar central frequencies. (b) Comparison of the corresponding temporal intensity profiles. 
Figure 1
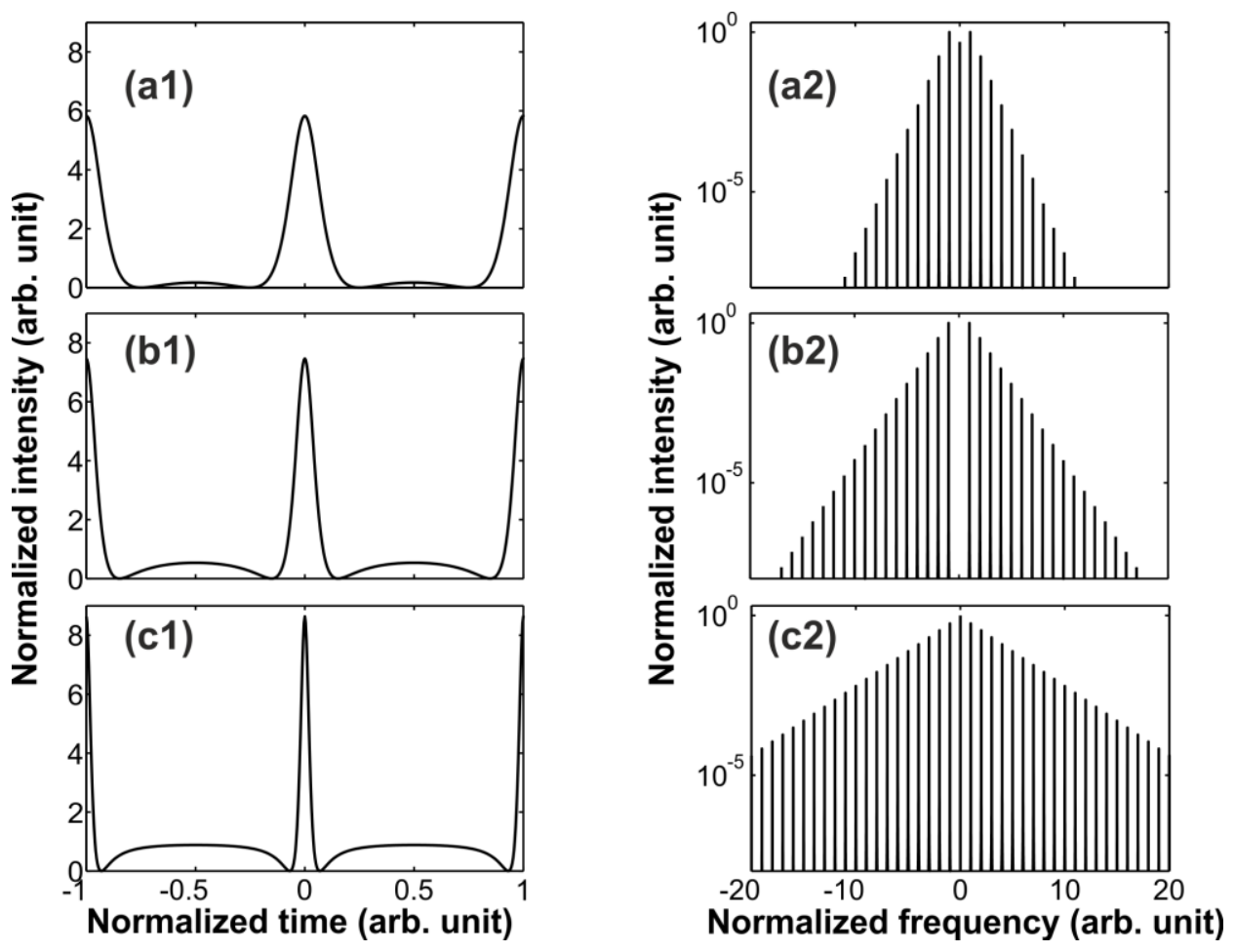
Figure 2

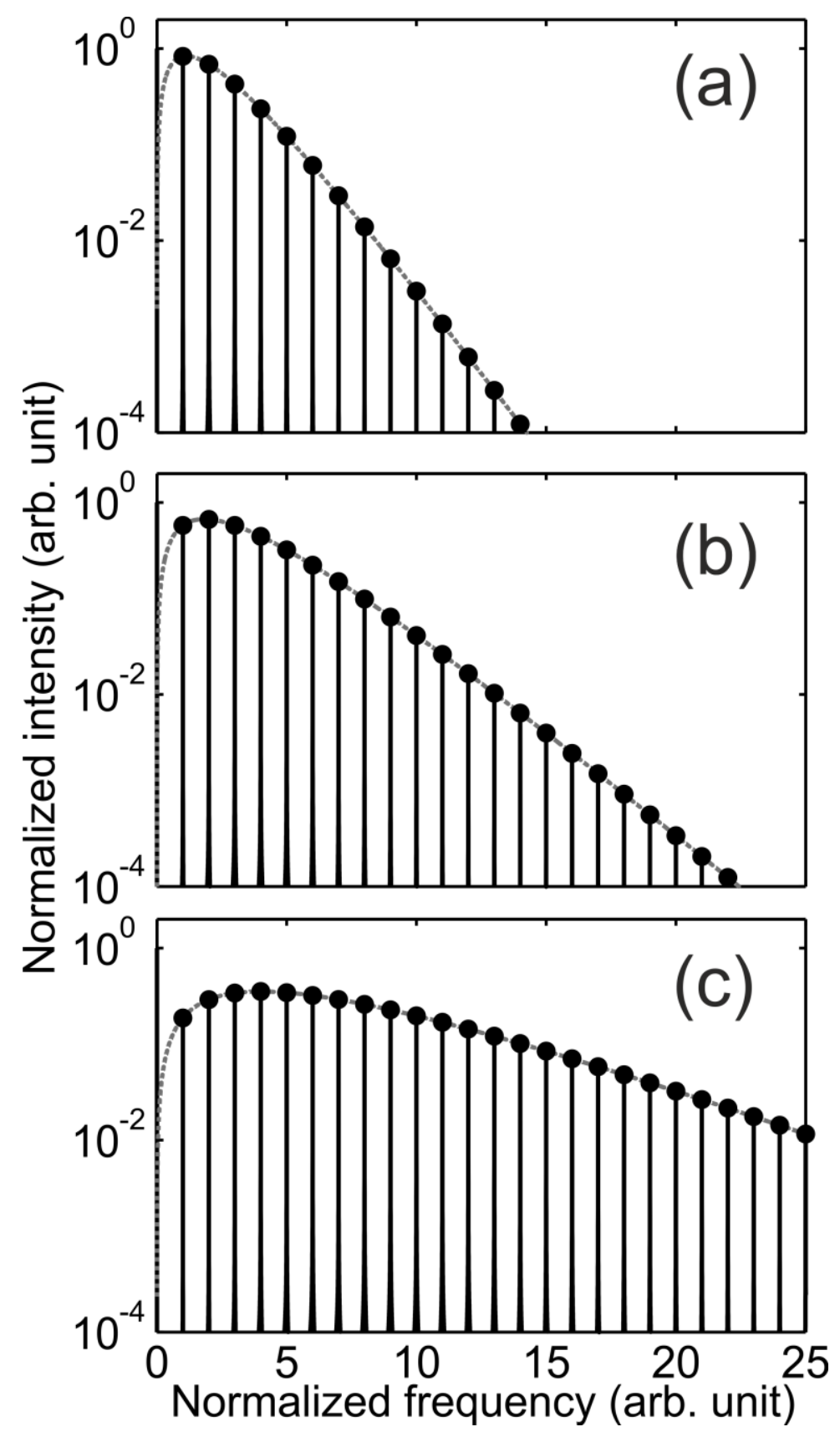


Figure 3
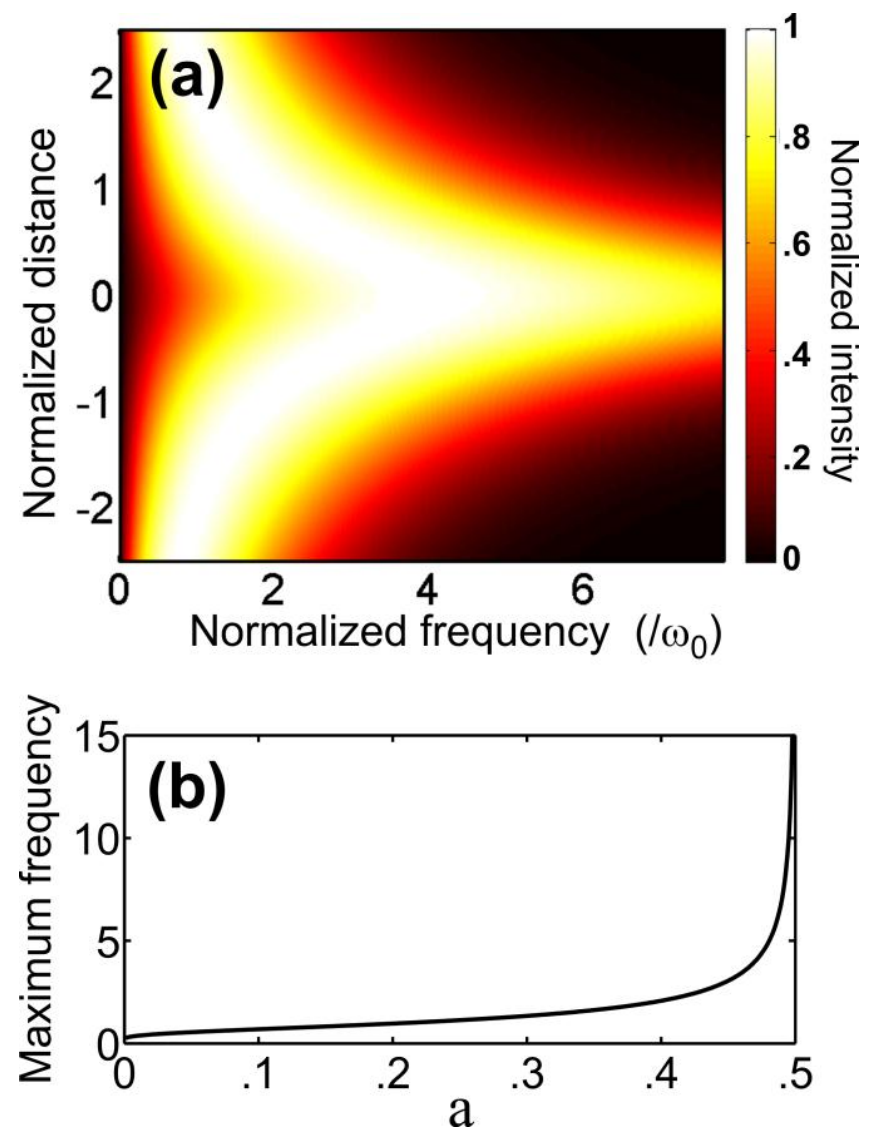
Figure 4
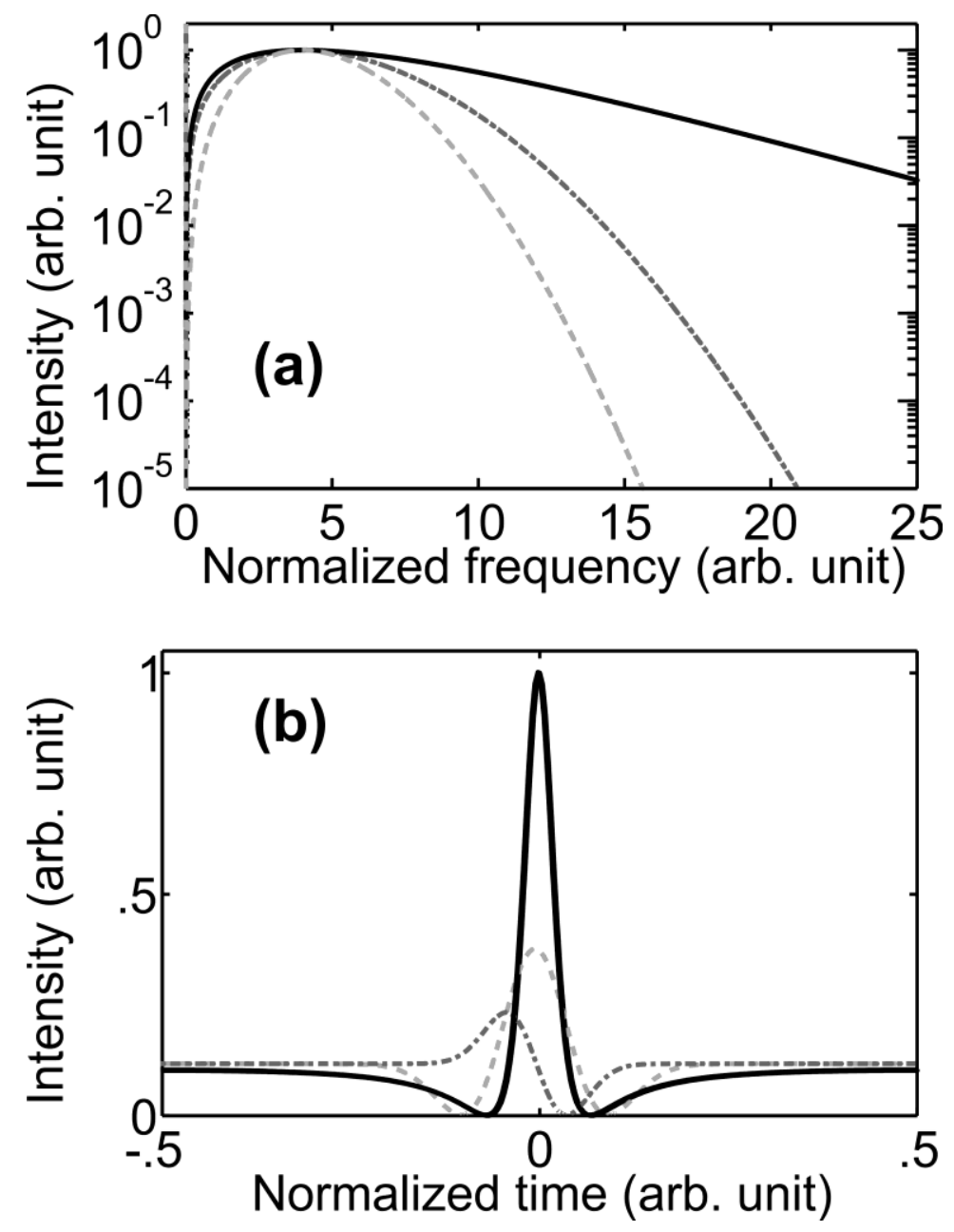


\section{References}

[1] E.A. Kuznetsov, Solitons in a parametrically unstable plasma, Doklady Akamemii Nauk SSSR, 22 (1977) 507-508.

[2] N.N. Akhmediev, V.I. Korneev, Modulation instability and periodic-solutions of the nonlinear Schrödinger equation, Theor. Math. Phys., 69 (1986) 10891093.

[3] N. Akhmediev, A. Ankiewicz, M. Taki, Waves that appear from nowhere and disappear without a trace, Phys. Lett. A, 373 (2009) 675-678.

[4] M. Onorato, D. Proment, G. Clauss, M. Klein, Rogue Waves: From Nonlinear Schrödinger Breather Solutions to Sea-Keeping Test, Plos One, 8 (2013) e54629.

[5] K. Hammani, B. Kibler, C. Finot, P. Morin, J. Fatome, J.M. Dudley, G. Millot, Peregrine soliton generation and breakup in standard telecommunications fiber, Opt. Lett., 36 (2011) 112-114.

[6] K. Hammani, B. Wetzel, B. Kibler, J. Fatome, C. Finot, G. Millot, N. Akhmediev, J.M. Dudley, Spectral dynamics of modulation instability described using Akhmediev breather theory, Opt. Lett., 36 (2011) 2140-2142.

[7] B. Kibler, J. Fatome, C. Finot, G. Millot, G. Genty, B. Wetzel, N. Akhmediev, F. Dias, J.M. Dudley, Observation of Kuznetsov-Ma soliton dynamics in optical fibre, Sci. Rep., 2 (2012) 463.

[8] M. Erkintalo, K. Hammani, B. Kibler, C. Finot, N. Akhmediev, J.M. Dudley, G. Genty, Higher-Order Modulation Instability in Nonlinear Fiber Optics, Phys. Rev. Lett., 107 (2011) 253901.

[9] J.M. Dudley, G. Genty, F. Dias, B. Kibler, N. Akhmediev, Modulation instability, Akhmediev Breathers and continuous wave supercontinuum generation, Opt. Express, 17 (2009) 21497-21508.

[10] C. Mahnke, F. Mitschke, Possibility of an Akhmediev breather decaying into solitons, Physical Review A, 85 (2012) 033808.

[11] J. Fatome, B. Kibler, C. Finot, High-quality optical pulse train generator based on solitons on finite background, Opt. Lett., 38 (2013) 1663-1665.

[12] N. Devine, A. Ankiewicz, G. Genty, J. Dudley, N. Akhmediev, Recurrence phase shift in Fermi-Pasta-Ulam nonlinear dynamics, Phys. Lett. A, (2011). 
[13] G. Van Simaeys, P. Emplit, M. Haelterman, Experimental Demonstration of the Fermi-Pasta-Ulam Recurrence in a Modulationally Unstable Optical Wave, Phys. Rev. Lett., 87 (2001) 033902.

[14] Y. Jianping, Z. Fei, W. Qing, Photonic Generation of Ultrawideband Signals, J. Lightw. Technol., 25 (2007) 3219-3235.

[15] A.B. Matsko, A.A. Savchenkov, L. Maleki, On excitation of breather solitons in an optical microresonator, Opt. Lett., 37 (2012) 4856-4858.

[16] K. Hammani, B. Kibler, J. Fatome, S. Boscolo, G. Genty, J.M. Dudley, G. Millot, C. Finot, Nonlinear spectral shaping and optical rogue events in fiberbased systems, Opt. Fiber. Technol., 18 (2012) 248-256. 